\title{
RNA-seq analyses of gene expression in the microsclerotia of Verticillium dahliae
}

\author{
Dechassa Duressa', Amy Anchieta', Dongquan Chen², Anna Klimes ${ }^{3,4,5}$, Maria D Garcia-Pedrajas ${ }^{6}$, \\ Katherine F Dobinson ${ }^{3,4}$ and Steven J Klosterman ${ }^{1 *}$
}

\begin{abstract}
Background: The soilborne fungus, Verticillium dahliae, causes Verticillium wilt disease in plants. Verticillium wilt is difficult to control since $V$. dahliae is capable of persisting in the soil for 10 to 15 years as melanized microsclerotia, rendering crop rotation strategies for disease control ineffective. Microsclerotia of $V$. dahliae overwinter and germinate to produce infectious hyphae that give rise to primary infections. Consequently, microsclerotia formation, maintenance, and germination are critically important processes in the disease cycle of $V$. dahliae.

Results: To shed additional light on the molecular processes that contribute to microsclerotia biogenesis and melanin synthesis in $V$. dahliae, three replicate RNA-seq libraries were prepared from 10 day-old microsclerotia (MS)-producing cultures of $V$. dahliae, strain VdLs.17 (average $=52.23$ million reads), and those not producing microsclerotia (NoMS, average $=50.58$ million reads). Analyses of these libraries for differential gene expression revealed over 200 differentially expressed genes, including up-regulation of melanogenesis-associated genes tetrahydroxynaphthalene reductase (344-fold increase) and scytalone dehydratase (231-fold increase), and additional genes located in a 48.8 kilobase melanin biosynthetic gene cluster of strain VdLs.17. Nearly $50 \%$ of the genes identified as differentially expressed in the MS library encode hypothetical proteins. Additional comparative analyses of gene expression in $V$. dahliae, under growth conditions that promote or preclude microsclerotial development, were conducted using a microarray approach with RNA derived from $V$. dahliae strain Dvd-T5, and from the amicrosclerotial $v d h 1$ strain. Differential expression of selected genes observed by RNA-seq or microarray analysis was confirmed using RT-qPCR or Northern hybridizations.

Conclusion: Collectively, the data acquired from these investigations provide additional insight into gene expression and molecular processes that occur during MS biogenesis and maturation in $\mathrm{V}$. dahliae. The identified gene products could therefore potentially represent new targets for disease control through prevention of survival structure development.
\end{abstract}

Keywords: Verticillium dahliae, Morphogenesis, Microsclerotia, RNA-seq, Gene expression

\section{Background}

Verticillium dahliae is a soilborne, plant pathogenic fungus that causes wilt disease on over 200 plant species worldwide. Verticillium wilt diseases are also referred to as vascular wilts since the fungus invades the plant xylem, disrupting water transport and causing characteristic leaf wilt symptoms. Control of Verticillium wilt diseases is complicated by the lack of genetic resistance in

\footnotetext{
* Correspondence: Steve.Klosterman@ars.usda.gov

'United States Department of Agriculture - Agricultural Research Service, Salinas, CA, USA

Full list of author information is available at the end of the article
}

many plant hosts, and also by the persistence of $V$. dahliae in the soil [1].

Verticillium dahliae produces melanized survival structures, microsclerotia, that are able to survive in the soil for at least 10 years $[2,3]$. Root exudates provide a signal for microsclerotial germination $[4,5]$, and upon germination infectious hyphae emerge from the microsclerotia to initiate plant root infection. Following penetration through the plant root epidermis, and an extended period of colonization in which plants are asymptomatic, microsclerotia are produced in large quantities within the dying and necrotic plant tissues, and are subsequently returned to the 
soil with plant debris to initiate a new round of the disease cycle [1].

Wild-type microsclerotia of $V$. dahliae are characterized by the presence of dense black melanin deposits that appear as granules within and exterior to the cell wall [6]. This melanin is proposed to act as an anti-desiccant and protect against temperature extremes and enzymatic lysis [6], and is synthesized via a dihydroxynaphthalene (DHN) melanin biosynthesis pathway [6,7]. The initial substrate molecule in the DHN pathway is 1,3,6,8-tetrahydroxynaphthalene (1,3,6,8-THN), derived by cyclization of acetate molecules by polyketide synthase (PKS) activity. DHN monomers are generated from the $1,3,6,8$-THN through alternating reduction and dehydration reactions involving several intermediates, including scytalone, 1,3,8-THN (trihydroxynaphthalene), and vermelone [6]. The DHN monomers are subsequently oxidized and polymerized into melanin by laccases $[6,8]$. Though the microsclerotia are typically darkly pigmented, the processes of melanin production and microsclerotial development in $V$. dahliae can be uncoupled [9].

Because of their central role in pathogen survival and initiating plant root infection, the microsclerotia of $V$. dahliae have been considered important targets for disease control $[10,11]$. This suggestion is supported by the correlation between reduced microsclerotial and pigment production, and reduced survival of $V$. dahliae $[12,13]$. The results of the analyses of multiple Agrobacterium tumefaciens-mediated transformation (ATMT) insertional mutants of $V$. dahliae also indicate an important link between microsclerotial formation and developmental processes needed for virulence [14,15]. For example, a strain of $V$. dahliae with mutation of the stress-responsive glutamic acid-rich protein encoding-gene (VdGarp1; VDAG_08781) shows both decreased virulence on cotton, and severely reduced microsclerotia formation under nutrient limiting conditions [14]. In contrast, however, $v d p k a C 1$ mutants exhibit both reduced virulence, and under certain growth conditions enhanced microsclerotia production [16]. The $v d h 1$ mutant, which exhibits an amicrosclerotial phenotype under both nutrient-rich and starvation growth conditions, is not compromised in virulence [17]. Taken together, these data indicate that microsclerotial development is not always coupled with virulence.

Analyses of gene expression in Verticillium dahliae during microsclerotia formation have been carried out previously by comparisons of expressed sequence tag (EST) libraries generated from $V$. dahliae cultures in which microsclerotia were developing (DMS), and those generated from cultures grown in simulated xylem fluid medium (SXM), which does not stimulate microsclerotia development [18]. Those EST analyses revealed in the DMS library expression of pigment synthesis genes, and increased expression of transporter type proteins encoded by several SXM library genes. With the availability of the genomic sequence and the transcriptome of strain VdLs.17 of $V$. dahliae via the Broad Institute [19], more in-depth analysis of gene expression during microsclerotia formation in $V$. dahliae can be conducted using the highthroughput, deep coverage RNA sequencing (RNA-seq) technology. As an additional resource, bioinformatic analyses have enabled categorization of gene sets in $V$. dahliae, strain VdLs.17 [19]. These gene sets include sequences encoding predicted secreted proteins, secondary metabolite synthesis genes, cysteine-rich proteins, and a set of 354 proteins encoded in four $\sim 300 \mathrm{~kb}$ lineage (VdLs.17)-specific "genome islands", that showed a significantly increased rate of expression relative to the core genome sequence [19]. All of these individually categorized gene sets can be useful for rapid data-mining in projects employing high throughput analyses of gene expression.

The aim of this study was to identify genes that are differentially expressed in the melanized microsclerotia (MS) of $V$. dahliae. To accomplish this aim, RNA-seq libraries were generated from MS-producing $(\mathrm{MS}+)$ cultures of strain VdLs.17, and from cultures of VdLs.17 not producing MS (NoMS), and analyzed for differential gene expression. Additional comparative analyses of gene expression, under growth conditions that promote or preclude microsclerotial development, were conducted using a microarray approach, with RNA derived from $V$. dahliae strain Dvd-T5 and an amicrosclerotial mutant ( $v d h 1$ strain) derived from Dvd-T5 by targeted mutagenesis [20]. Taken together, these results yielded additional insights into gene sets that are of likely importance to the processes involved in melanin production in $V$. dahliae, as well as in MS formation and maintenance, or inhibition of these processes.

\section{Results}

\section{RNA-seq analyses}

Microsclerotia formation in Verticillium dahliae is a default process that occurs as cultures age, and on the potato dextrose agar (PDA) used for the RNA-seq analysis is typically initiated after 3-4 days growth. However, during our studies of $V$. dahliae we have noted that on occasion cultures may spontaneously become amicrosclerotial (K.F. Dobinson, S.J. Klosterman, unpublished). We considered this phenomenon as an opportunity that could be exploited for the analysis of microsclerotial development. Two different RNA-seq approaches were therefore used to assess differential gene expression between MS + and NoMS PDA-grown cultures (Figure 1 ) of $V$. dahliae. In the first, a genome-wide analysis (GWA) was performed using untrimmed RNA-seq library sequences and FDR-corrected P values (see Methods), and differences were evaluated in gene expression between MS + and NoMS libraries. This method detected a total of 145 differentially expressed genes, 64 of which were up-regulated, and 81 down- 


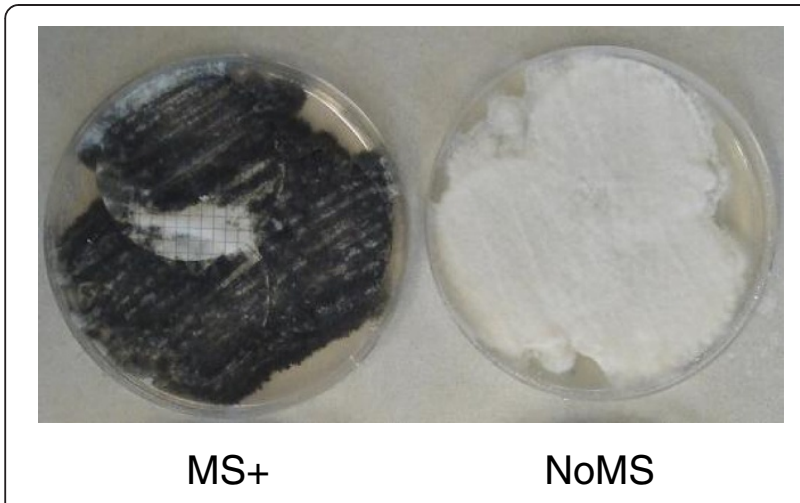

Figure 1 Ten day-old cultures of Verticillium dahliae strain VdLs17, that produced microsclerotia (MS+) or did not produce microsclerotia (NoMS). Both cultures were grown on potato dextrose agar overlaid with nitrocellulose membranes and incubated at $25^{\circ} \mathrm{C}$.

regulated in the MS + versus NoMS cultures (Additional files 1 and 2). GWA was also carried out using trimmed RNA sequence reads without applying FDR correction to $\mathrm{P}$ values, and selecting genes having $>10$-fold up- or down-regulation (listed in Additional file 3). This latter approach resulted in the detection of increased numbers of up-regulated genes involved in melanin synthesis (Additional file 4), a hallmark feature of MS maturation [6]. Specifically, nine pigment synthesis genes related to fungal melanin biosynthesis were identified by data mining analysis (DMA) as up-regulated in the MS + library vs. NoMS library (Additional file 4, \#s 1-9), whereas only two of these genes were detected with the first GWA (Additional file 1, \#s 1-2). The results of this second GWA (GWA2) were therefore a focal point for this study and were combined into the DMA (Additional file 3) as described below.

The DMA was conducted with 12 sets of genes of interest (GOIs) (Additional file 3). One set of genes examined in this analysis was created by inclusion of the selected genes from GWA2 having > 10-fold up- or down-regulation (Additional file 3). The other 11 gene sets were obtained either from categories of $V$. dahliae genes identified in previous analyses [19], or from multigene families (such as PKSs, NRPSs, hydrophobins, and THN reductases) identified in this study as containing differentially expressed genes (Table 1; Additional file 3). All genes in each set were analyzed for differential expression between the MS + and NoMS libraries, using 10-base end-trimmed RNA sequence reads, and employing common significance criteria of FDR-corrected $P$ values $<0.05$, and expression fold change of at least 1.5-fold up- or down-regulation. This analysis yielded a total of 210 differentially expressed genes, 153 of which were up-regulated (Additional file 4) and 57 downregulated (Additional file 5). Those genes with $>20$ fold changes in differential expression are shown in Table 2.

In many instances, the recorded magnitude of gene expression fold-change was greater in the DMA than in the GWA (Additional file 6). In DMA, the largest foldchange among the up-regulated and down-regulated genes in the MS + library was recorded for VDAG_03665 (encoding THN reductase, 344-fold, Table 2), and VDAG_03287 (encoding hypothetical protein, Table 2), respectively. The DMA also enabled detection of additional pigment synthesis genes expected to be identified in the MS + library, as indicated above.

The number of up- and down-regulated genes observed in DMA of the various categories of $V$. dahliae

Table 1 Differential gene expression counts for data mining analyses of the Verticillium dahliae genome

\begin{tabular}{|c|c|c|c|c|}
\hline \multirow[t]{2}{*}{ Gene category } & \multirow{2}{*}{$\begin{array}{c}\text { Total } \\
\text { number } \\
\text { in } V . \\
\text { dahliae } \\
\text { genome }\end{array}$} & \multicolumn{3}{|c|}{ Differentially expressed* } \\
\hline & & Upregulated & Downregulated & Total \\
\hline Hydrophobins & 5 & 0 & 3 & 3 \\
\hline Tetrahydroxynapthalene reductase & 3 & 3 & 0 & 3 \\
\hline Vascular wilt specific genes & 14 & 4 & 0 & 4 \\
\hline Cytochrome P450s & 69 & 3 & 0 & 3 \\
\hline LS region genes & 354 & 41 & 6 & 47 \\
\hline$A B C$ type transporters & 35 & 4 & 1 & 5 \\
\hline MFS type transporters & 272 & 13 & 2 & 15 \\
\hline Predicted secretome & 780 & 40 & 33 & 73 \\
\hline Polyketide synthases & 11 & 2 & 0 & 2 \\
\hline Small cysteine rich proteins & 258 & 14 & 11 & 25 \\
\hline NRPSS & 8 & 0 & 0 & 0 \\
\hline
\end{tabular}

*Differential expression between MS + and NoMS libraries. 
Table 2 Genes $>20$ fold up- or down-regulated in microsclerotia forming (MS +) vs non microsclerotia forming (NoMS) cultures of Verticillium dahliae as revealed by data mining analysis of RNA-seq data

\begin{tabular}{ll}
\hline Functional category/gene ID & Expressio \\
\hline Pigment synthesis & \\
VDAG_03665 & 344.05 \\
VDAG_03393 & 231.29 \\
VDAG_04954 & 165.00 \\
VDAG_00190 & 136.66 \\
VDAG_00189 & 110.81 \\
VDAG_05181 & 86.98 \\
VDAG_00183 & 40.79 \\
VDAG_00184 & 23.13
\end{tabular}

\section{General metabolism}

$\begin{array}{ll}\text { VDAG_03650 } & 246.84 \\ \text { VDAG_03079 } & 60.61 \\ \text { VDAG_09583 } & 20.18\end{array}$

\section{Transporters}

VDAG_02154

Cytoskeleton/cell adhesion

VDAG_04170

Hypothetical proteins

$\begin{array}{ll}\text { VDAG_01806 } & 251.76 \\ \text { VDAG_00621 } & 201.14 \\ \text { VDAG_03732 } & 97.43 \\ \text { VDAG_02042 } & 67.51 \\ \text { VDAG_03078 } & 60.72 \\ \text { VDAG_07349 } & 58.42 \\ \text { VDAG_02389 } & 42.48 \\ \text { VDAG_08973 } & 37.13 \\ \text { VDAG_05569 } & 30.02 \\ \text { VDAG_05179 } & 29.71 \\ \text { VDAG_10456 } & 28.13 \\ \text { VDAG_00592 } & 27.17 \\ \text { VDAG_04171 } & 24.46 \\ \text { VDAG_06885 } & 22.53 \\ \text { VDAG_09869 } & 20.88 \\ \text { VDAG_00490 } & 20.38\end{array}$

\section{Cell death}

VDAG_00261

$$
72.35
$$

IDI-3/induced during incompatibility/cell death
Functional category/gene ID Expression fold-change* (down-regulated genes) General metabolism

$\begin{array}{ll}\text { VDAG_02162 } & 46.09 \\ \text { VDAG_03507 } & 39.98 \\ \text { VDAG_04322 } & 31.66\end{array}$

Tetrahydroxynaphthalene reductase/melanin biosynthesis

Scytalone dehydratase/melanin biosynthesis

Pigment biosynthesis protein Ayg1

Conidial yellow pigment biosynthesis polyketide synthase/ melanin synthesis

Laccase/melanin biosynthesis

Tetrahydroxynaphthalene reductase/melanin biosynthesis

Versicolorin reductase/Polyketide/melanin or aflatoxin biosynthesis

Amino acid adenylation/polyketide synthase

Cytochrome P450 2C3/oxidizes steroids, fatty acids, xenobiotics

Catalase/involved in oxidative stress relief

Alcohol oxidase

RTA1/lipid translocating exporter/drug resistance protein

Keratin associated protein-10

Unknown
Unknown
Unknown
Unknown
Unknown
Unknown
Unknown
Unknown
Unknown
Unknown
Unknown
Unknown
Unknown
Unknown
Unknown
Unknown

Protein name/functional annotation

Oviduct spcefic glycoprotein/glycosyl hydrolase family

Aldo-keto reductase/yakc

Acetylcholinesterase/hydrolyze acetylcholine 
Table 2 Genes $>20$ fold up- or down-regulated in microsclerotia forming (MS +) vs non microsclerotia forming (NoMS) cultures of Verticillium dahliae as revealed by data mining analysis of RNA-seq data (Continued)

\begin{tabular}{lll}
\hline VDAG_08741 & 29.50 & Endochitinase \\
VDAG_06138 & 24.36 & 6-hydroxy-D-nicotine oxidase \\
Hypothetical proteins & & \\
VDAG_03287 & 272.06 & Unknown \\
VDAG_03216 & 42.95 & Unknown \\
VDAG_03585 & 38.51 & Unknown \\
VDAG_04032 & 36.48 & Unknown \\
\hline
\end{tabular}

*FDR $P$ value $<0.05$ and fold change $>1.5$.

genes is summarized in Table 1. Among the 11 categories, predicted secretome, LS region protein-encoding genes, and small cysteine-rich protein-encoding genes represented the largest fractions of genes differentially expressed between the two libraries. Functional classification of the differentially expressed genes detected with DMA revealed that over half encode hypothetical proteins of unknown function, with proteins involved in cell metabolism and transport functions representing the second and third largest category, respectively (Additional file 7).

\section{Functional categories of differentially expressed genes}

The lists of genes identified as differentially expressed during microsclerotia development were functionally annotated in-silico to identify genes encoding the different types of proteins that are potentially involved in microsclerotia biogenesis. Among these were genes coding for pigment biosynthesis and secondary metabolic enzymes, as well as those coding for cell growth, morphogenesis, signaling and transcription factors associated with pigment production and/or nutrient acquisition, and carbohydrate active enzymes and transport proteins. Below we describe in more detail some of the genes identified as differentially expressed in each one of these major categories.

\section{Pigment synthesis and secondary metabolism}

A number of known melanogenesis-related enzymeencoding genes were identified by the data mining analysis as up-regulated in MS + culture vs. the NoMS culture, including THN reductase (VDAG_03665, 344-fold; $V D A G \_05181$ 87-fold), scytalone dehydratase (VDAG_ 03393, 231-fold), pigment biosynthesis protein Ayg1 (VDAG_04954, 165-fold), conidial yellow pigment biosynthesis PKS (VDAG_00190, 137-fold), two laccases (VDAG_00189, 111-fold, and VDAG_00034, 7-fold), versicolorin reductase (VDAG_00183, 41-fold), polyketide synthase (VDAG_00184, 23-fold), (Table 2, Additional file 4, \#1-9). Among these, those encoding the PKS (VDAG_00190), versicolorin reductase (VDAG_00183), laccase (VDAG_00189) and a second polyketide synthase (VDAG_00184) are found clustered in a region spanning $48.8 \mathrm{~kb}$ on chromosome 2, supercontig 1 of the $V$. dahliae strain VdLs.17 genome (http://www. broadinstitute.org/annotation/genome/verticillium_dahliae) (Figure 2).

Microsclerotia formation in fungi involves dramatic morphological changes and activation of biosynthetic pathways involved in pigment synthesis [21], processes that are expected to entail transcriptional activation of genes of various cellular functions. Consistent with this premise, several transcriptional regulators were observed as upregulated in the DMA, including some that are known to control melanin biosynthesis (Table 2, Additional file 4, \#47-49). Homologs of the Pig1 and CMR1 transcription factors, which have been previously reported to be involved in fungal melanin synthesis [22,23], were both upregulated 12-fold in V. dahliae VdLs.17 (Additional file 4, \#47 and \#48). These genes share sequence homology, and are located next to each other in the aforementioned $48.8 \mathrm{~kb}$ gene cluster (Figure 2). In addition, a novel transcription factor, encoded by VDAG_00192 and located in the same cluster, was transcriptionally activated in the MS + culture. The Pig1 and CMR1 homologs were originally annotated in the Verticillium genome sequence (Broad Institute) as two separate genes (VDAG_00194 and $\left.V D A G \_00195\right)$, but are probably encoded by a single gene (Figure 2). This possibility is supported by the finding in the current study of nearly identical RNA-seq expression levels of VDAG_00194 and VDAG_00195 (Additional file 3).

Secondary metabolite biosynthesis in fungi is tightly linked with functions of non-ribosomal peptide synthetases (NRPSs), PKSs, and activities of the cytochrome p450 enzymes (CYPs). Of the eight NRPSs identified in the $V$. dahliae genome (see Methods), none were significantly differentially expressed in the MS + vs. NoMS RNA-seq libraries, whereas of the 10 PKS-encoding genes identified in this species, only the two above-mentioned, VDAG_00184 and VDAG_00190, which share homology with fungal genes involved in the secondary metabolic pathway leading to pigment production [24], and are part of the melanin biosynthesis cluster (Figure 2), were detected as differentially expressed. Also, 3/69 of the CYPs were up-regulated in the MS + library (Table 1). Expression of the CYP gene 


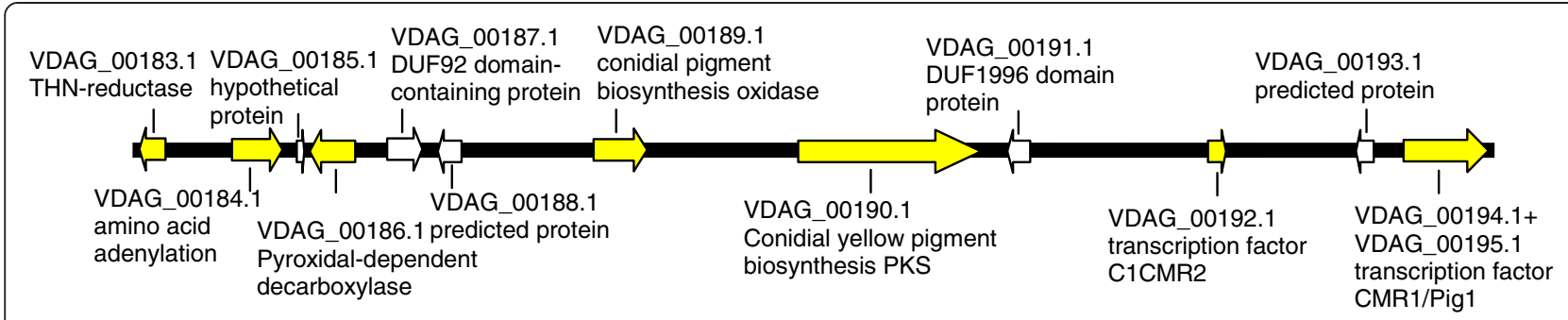

$2.0 \mathrm{~kb}$

Figure 2 A 48.8 kb melanin biosynthetic cluster of genes in Verticillium dahliae. Genes up-regulated in the MS + culture are highlighted in yellow.

VDAG_03650 was up-regulated 247-fold in the MS + library (Table 2, Additional file 4, \#15), and that of VDAG_08399 was up-regulated 5-fold (Additional file 4, \#40). Though its function in $V$. dahliae is currently unknown, BLAST searches revealed that VDAG_08399 shares homology with $\mathrm{O}$-methylsterigmatocystin oxidoreductase proteins involved in the conversion of $\mathrm{O}$-methylsterigmatocystin to aflatoxins in Aspergillus spp. [25].

Cell growth, morphogenesis and cell death-related genes A homolog of IDI-3, a cell death-associated gene of Podospora anserina [26,27], was strongly up-regulated (72fold) in the MS + culture (Table 2, Additional file 4, \#153), as were cell cycle/cytoskeleton-related genes. The keratinassociated protein-10 gene homolog (VDAG_04170; upregulated 23-fold, Table 2, \#70) encodes a large (2924 amino acids) cysteine-rich protein, predicted to be extracellular [19] and displaying weak homology with the developmentally regulated conidiospore surface protein Cmp1, from Trichoderma sp. [28]. Genes encoding homologs of actin and other cytoskeletal elements that play critical roles in fungal morphogenesis [29] were also differentially expressed in the MS + or NoMS cultures. A gene encoding a homolog of the cortical actin regulatory protein, Asp1, important for polarized cell growth [30], was up-regulated 4-fold in the MS + culture (Additional file 4, \#71). There was also down-regulation of other cytoskeletal-related protein genes (Additional file 5, \#s 41-43), and of a homolog of SUN4 (Additional file 5, \#27), which in S. cerevisiae regulates cell wall morphogenesis and septation [31].

Catalases, whose products mediate the conversion of hydrogen peroxide to $\mathrm{O}^{-}$and $\mathrm{H}_{2} \mathrm{O}$, have been implicated in oxidative stress protection [32], and in mediating sclerotial differentiation in filamentous fungi [33]. The expression of only one of three genes encoding predicted secreted catalases, VDAG_03079, was up-regulated 61fold in the MS + culture (Table 2, Additional file 4, \#16), while the others (VDAG_04826 and VDAG_06575) were neither significantly up- nor down-regulated (Additional file 3).

We observed a 2-fold increase in the expression level of VdpkaC1 (Additional file 1, \# 31; VDAG_06474), which encodes the catalytic subunit of a cAMP-dependent kinase that is involved in regulation of microsclerotial development [16].

\section{Carbohydrate-active enzymes}

The genome of $V$. dahliae encodes an abundance of carbohydrate-active enzymes (CAZys) with diverse predicted activities [19], including roles in fungal cell wall remodeling and plant cell wall degradation. CAZyencoding sequences significantly up-regulated in the MS library included protein-encoding genes endochitinase VDAG_04833 (Additional file 4, \#42), pectate lyase VDAG_05402 (Additional file 4, \#36), alpha-amylase A VDAG_05976 (Additional file 4, \#37), glucan 1,3-betaglucosidase VDAG_09744 (Additional file 4, \#29), glucanase B VDAG_10470 (Additional file 4, \#31), and chitin deacetylase VDAG_05660 (Additional file 4, \#25). CAZy genes significantly down-regulated included those encoding an alpha-galactosidase A VDAG_02431 (Additional file 5, \#19), a glucan 1,3-beta-glucosidase VDAG_07185 (Additional file 5, \#21), endoglucanase VDAG_06254 (Additional file 5, \#17), glucan endo-1,3-alpha-glucosidase (VDAG_04101, 14-fold), and an endochitinase $V D A G \_08741$ (Additional file 5, \#9). The periplasmic trehalase VDAG_03038 and the glucose-repressive Grg1 [34] gene homolog VDAG_01467 were also up-regulated 10- and 13-fold (Additional file 4, \#34 and \#73, respectively).

\section{Transport proteins}

The data-mining analyses revealed differential expression in the MS + vs. NoMS libraries of genes encoding ATP-binding cassette (ABC) and major facilitator (MFS) transport proteins, and other transporters. In total, 4/35 $\mathrm{ABC}$ type proteins were differentially regulated in the 
MS library, while 15/272 MFS proteins were differentially regulated (Table 1 ). Of the differentially expressed $A B C$ transporter-encoding genes, the observed magnitudes of the differences in expression were relatively small, from 2.5 -fold up- to 2-fold down-regulation. Only one of the $\mathrm{ABC}$ type and two of the predicted MFS genes were identified as down-regulated (Table 1). The sugar transport MFS protein-encoding gene VDAG_08124 (Additional file 4, \#53), was strongly induced, with a 17 -fold change in expression. Of the additional transporter type genes identified, the RTA1/lipid translocating exporter gene VDAG_02154 (Table 2, Additional file 4, \#51), was upregulated 31-fold. The sphingolipid long-chain baseresponse protein (PIL1) gene (VDAG_04085), involved in endocytic transport regulation [35], was also up-regulated 12-fold (Additional file 4, \#54).

\section{Hypothetical protein-encoding genes}

Approximately $50 \%$ of the differentially expressed genes in the MS + library encode hypothetical proteins for which cellular functions are not yet assigned (Additional file 7). Some of these genes were strongly induced in the MS + library with expression fold changes $>50$ (Table 2). VDAG_01806, up-regulated 252-fold in the MS + library, was also identified as an EST (VD0100E03) in the library derived from a developing microsclerotia (DMS) culture of $V$. dahliae $([17,18]$. At the other end of the spectrum, VDAG_03287 represents one of the strongly down-regulated (272-fold) hypothetical protein-encoding genes in the MS library (Additional file 5, \#29).

Protein motif searches (see Methods) were done for each of the hypothetical proteins in a quest for additional functional information. Most of the hypothetical protein encoding genes appeared to have no identifiable domain in protein motif searches, while others possess domains that function in diverse cellular activities, such as a fasciclin (FAS1) extracellular cell adhesion domain (Additional file 4, \#84), a cAMP-regulated phosphoprotein/endosulfineconserved region (Additional file 4, \#111) which might be involved in cell signaling, and fungal $\mathrm{Zn}(2)-\mathrm{Cys}(6)$ binuclear cluster (Additional file 4, \#121 and \#124) or bZIP domains (Additional file 4, \#127) with potential roles in transcription regulation.

\section{Reverse-transcription quantitative PCR (RT-qPCR) analyses of gene expression in MS and NoMS cultures}

Real-time PCR with Taqman assays were conducted to confirm differential expression of seven selected genes in MS + and NoMS cultures of $V$. dahliae (different culture types selected for this purpose are shown in Figure 3). These seven genes included known and hypothetical protein coding genes, as indicated in Figure 4. Among these, RNA-seq analyses had revealed that expression of the hypothetical protein encoding gene VDAG_03287 was strongly down-regulated in the MS + culture as compared to the NoMS culture. The six other genes in this set were up-regulated as determined by the RNAseq analyses (Additional file 4).

For the selected genes RT-qPCR analyses of relative expression was compared as follows: expression in the darkly pigmented 12 day MS + cultures $(12 \mathrm{~d} \mathrm{Ms}+)$ vs. that in the 12 day pure white NoMS (12 d NoMS) culture, and that in seven day-old cultures that showed an intermediate level of pigmentation ( $7 \mathrm{~d}$ MS +). Expression of the selected genes in darkly pigmented 6 month-old cultures (6 mo MS +) was also quantified and compared with expression in the above mentioned conditions (Figure 3). Culture types subjected to this study where pigment was either present or absent (Figure 3), were analyzed microscopically at each of the selected time points. Microscopic observations confirmed absence of structures resembling microsclerotia in those cultures lacking dark pigmentation (data not shown).

The results of the RT-qPCR analyses in $12 \mathrm{~d}$ MS + vs. $12 \mathrm{~d}$ NoMS cultures revealed significant up-regulation of VDAG_03650,VDAG_00621,VDAG_04954,VDAG_01806, and VDAG_08124 in the $12 \mathrm{~d}$ MS + culture, but showed no significant up- or down-regulation of VDAG_03287 or VDAG_05123 $(\mathrm{P}<0.05)$ (Figure 4). However, all of the genes tested that were significantly up-regulated in the MS culture, as determined by RNA-seq analyses, were also shown by RT-qPCR to be significantly upregulated in the 6 month MS + culture compared to that in the $12 \mathrm{~d}$ NoMS culture. Comparison of relative expression between the $6 \mathrm{mo}$ MS + culture and the $12 \mathrm{~d}$ NoMS culture types also revealed significant down-regulation of VDAG_03287 $(\mathrm{P}<0.05)$ in the former (Figure 4). With exceptions of VDAG_03287 or VDAG_05123 in the $12 \mathrm{~d}$ MS + vs. 12 d NoMS comparison, fold-changes in gene expression were similar as assessed by both RNA-seq and RTqPCR (Additional file 8).

As may be expected in the comparison of the expression levels in two $12 \mathrm{~d}$ and $7 \mathrm{~d}$ MS + cultures, differences in RT-qPCR-assessed relative gene expression were reduced for VDAG_00621,VDAG_04954,VDAG_01806, and VDAG_08124 (Figure 4). As mentioned, the $7 \mathrm{~d} \mathrm{MS}+$ cultures exhibited an intermediate level of melanization (Figure 3). Gene expression was markedly reduced for several of the genes when comparing the darkly pigmented 6 month MS + and $12 \mathrm{~d}$ MS + cultures. In particular, VDAG_03650,VDAG_03287, and the known pigment biosynthesis gene VDAG_04954 were significantly downregulated in the 6 month MS + culture, relative to the expression seen for the $12 \mathrm{~d}$ MS + culture (Figure 4).

\section{Microarray analyses}

In addition to the RNA-seq analyses done for MS + and NoMS cultures of $V$. dahliae strain VdLs.17, a small- 


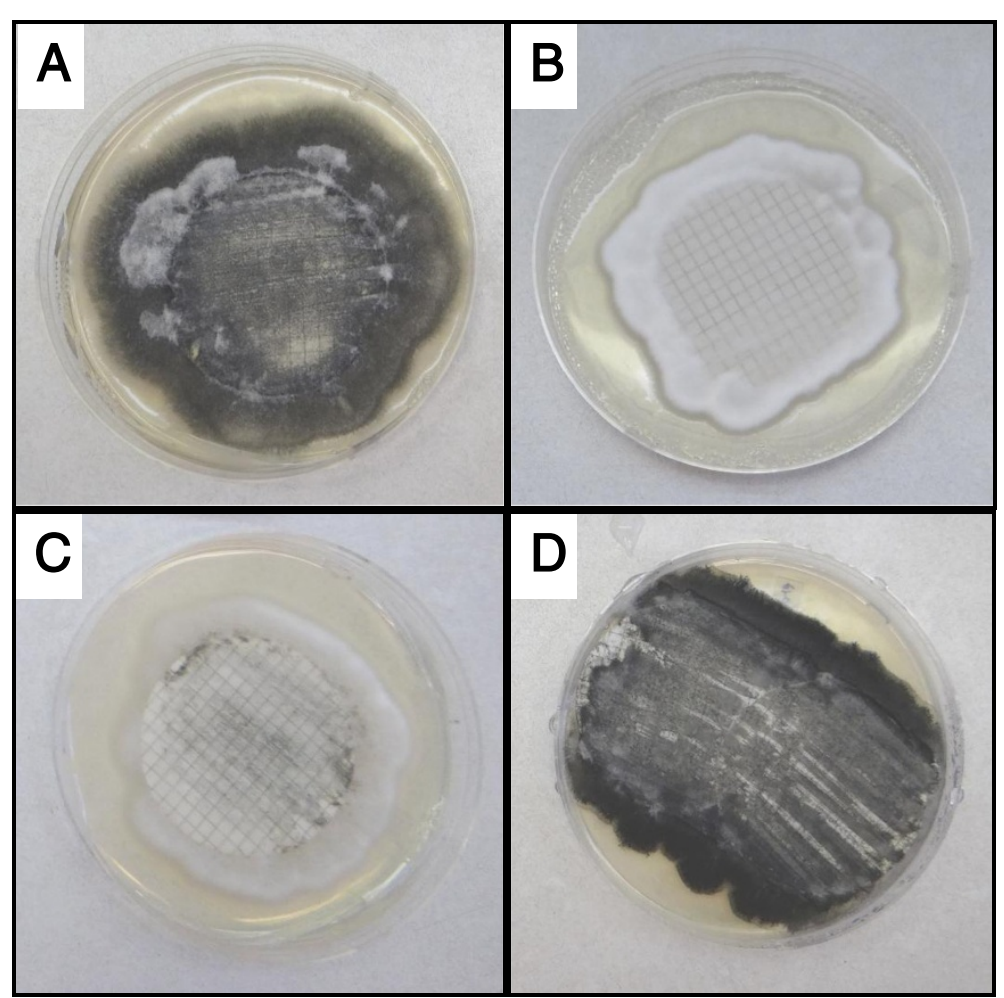

Figure 3 Examples of cultures of Verticillium dahliae that were used to determine relative gene expression by real-time quantitative PCR. A) A darkly pigmented 12 day culture of $V$. dahliae in which microsclerotia have formed (MS +). B) A 12 day culture of $V$. dahliae in which MS have not formed (NoMS). C) A 7 day culture of $V$. dahliae in which MS formation has occurred, but at an intermediate level relative to cultures $\mathrm{A}$ and $\mathrm{D}$ (note the slight pigmentation in C). Cultures $\mathrm{A}-\mathrm{C}$ were maintained at $25^{\circ} \mathrm{C}$. D) A culture stored for approximately 6 months at $4^{\circ} \mathrm{C}$ after an initial incubation period of 4 days at $25^{\circ} \mathrm{C}$. The gridded circular nitrocellulose membranes, which allowed easy harvest of the fungal MS and mycelia, are visible in A - C, but obscured in D.

scale microarray analysis was conducted in a second laboratory. V. dahliae strains and culture conditions used in the microarray and RNA-seq experiments is shown in Additional file 9. This microarray analysis used a second wild type strain, Dvd-T5, and an amiscrosclerotial $(v d h 1)$ strain, with cultures grown on either a complete medium agar (CMA), or a basal medium agar (BMA) that induces enhanced production of microsclerotia (Additional file 9).

In this analysis genes were identified that were induced during growth on BMA, and/or repressed in $v d h 1$ cultures, or Dvd-T5 cultures grown in a liquid CM, i.e. in the absence of microsclerotial development. Genes that exhibited such an expression profile belong to the aforementioned categories of genes found by RNA-seq analysis to be induced during microsclerotial development (Additional file 4) - namely, pigment biosynthesis and secondary metabolism genes, transcriptional regulators, and hypothetical genes. Normalized hybridization ratios of specific genes significantly induced during growth on BMA and/or repressed in the $v d h 1$ strain and during growth in liquid are shown in Table 3. Many of these genes are involved in secondary metabolism, including the following five pigment-biosynthesis protein-encoding genes that were also shown by the RNA-seq analyses to be up-regulated during microsclerotial development: scytalone dehydratase (VDAG_03393), versicolorin reductase (VDAG_00183), THN reductase (VDAG_03665), laccase (VDAG_00189), and Ayg1 (VDAG_04954) (Table 3). Also among the secondary metabolism genes that were up-regulated during growth in microsclerotia-promoting conditions in both the arrays and the RNA-seq analysis was the cytochrome p450 gene VDAG_03650 (Table 3).

Among hypothetical genes, two that were up-regulated in the RNA-seq analysis, VDAG_0713 and VDAG_01806, were also represented among the differentially expressed genes identified by the array analysis, with both shown to be induced during growth on BMA. Notably, the microarray analysis showed up-regulation of hypothetical gene VDAG_03287, which was, conversely, downregulated in the RNA-seq analysis (Table 2).

The microarray analysis also identified several differentially expressed genes in $V$. dahliae Dvd-T5 that were not identified in the RNA-seq analysis (Table 3.). Among these was the transcriptional regulator gene ACE1 (VD0110G09; VDAG_03150), which in Dvd-T5 was repressed in cultures 




Figure 4 Taqman-based reverse transcription-quantitative PCR (RT-qPCR) assays of relative gene expression in Verticillium dahliae. The seven genes selected for RT-qPCR experiments were identified by RNA-seq analyses as up- or down-regulated in cultures producing microsclerotia (MS +) or not producing microsclerotia (NoMS) at the sampling time points indicated. Mean expression and error was determined using the Relative Expression Software Tool [58] with ubiquitin and $\beta$-tubulin as reference genes (see Methods). Relative expression data from three biological replicates were examined. Error bars indicate 95\% confidence intervals. *Up-regulated genes $(\mathrm{P}<0.05)$. ${ }^{*}$ Down-regulated genes $(P<0.05)$.

that were not producing MS, and induced during MS development. A cellulose growth specific protein encoding gene (VDAG_04795), was also repressed under nonmicrosclerotial producing conditions, as were two genes with possible roles in lipid metabolism, an acetyl CoA synthetase gene (VDAG_09589), and a fatty acid binding protein-encoding gene (VD0103C06; VDAG_00137).

\section{Northern blot validation of microarray and RNA-seq data}

The expression of four genes, identified by RNA-seq as up-regulated in the MS + cultures of strain VdLs.17, and by microarray analyses in microsclerotial-producing cultures of strain Dvd-T5, was validated by Northern blot analyses (Additional file 10). These genes, which included two hypothetical protein encoding genes (VDAG_07138 and VDAG_01806) and two pigment biosynthesis genes (SCD1; VDAG_03393 and versicolorin reductase: VDAG_00183) were highly expressed at four days post-inoculation in wild-type cultures of $V$. dahliae grown on BMA, but suppressed in a $v d h 1$ mutant strain of $V$. dahliae grown on CMA (Table 3), and during growth of strain Dvd-T5 in liquid CM. Northern blot analysis also verified that the hypothetical gene VDAG_03287, which was down-regulated in the RNAseq analysis, was up-regulated in four-day old BMAgrown cultures (Additional file 10). The expression profiles of two genes, identified as differentially expressed in the microarray analysis but not in the VdLs.17 RNAseq analysis, verified by Northern analysis; both the ACE1 transcriptional regulator gene VDAG_03150 and the fatty acid binding protein VDAG_00137 were induced in Dvd$\mathrm{T} 5$, and repressed in both the $v d h 1$ mutant and in liquidgrown cultures (Additional file 10).

Ten genes were shown by microarray analysis to be strongly induced in strain Dvd-T5 during microsclerotia development. To evaluate inter-experimental results the expression of the genes as determined by microarray analysis was compared to that as assessed by Northern blot analysis, RNA-seq and RT-qPCR (Additional file 11). Of the ten genes, eight were also up-regulated in the MS + PDA cultures of strain VdLs.17 as compared to those in the RNA-seq analysis that were not producing MS on PDA (Additional file 11). The exceptions were $V D A G \_03287$, which was shown as down-regulated 
Table 3 Microarray analyses of Verticillium dahliae genes induced in a wild type strain and repressed in a hydrophobin mutant strain (vdh1)

\begin{tabular}{|c|c|c|c|c|c|c|}
\hline \multirow[t]{3}{*}{ EST ID } & \multirow{3}{*}{$\begin{array}{l}\text { EST match/ } \\
\text { comments }\end{array}$} & \multirow[t]{3}{*}{ Putative gene ID (BLASTX match) } & \multirow[t]{3}{*}{ RNA-seq ID } & \multirow{2}{*}{\multicolumn{3}{|c|}{$\begin{array}{c}\text { Expression ratios } \\
\text { Control }^{\mathrm{a}} \text { vs. }\end{array}$}} \\
\hline & & & & & & \\
\hline & & & & $\mathrm{vdh} 1^{\mathrm{b}}$ & $\mathrm{LIQ}^{\mathrm{c}}$ & $B M^{d}$ \\
\hline \multicolumn{7}{|c|}{ Genes induced in BM } \\
\hline VD0107B12 & BQ109916 VD0100E09 & SCD1 & VDAG_03393 & $0.22^{*}$ & $0.22^{*}$ & $9.42^{* *}$ \\
\hline VD0107A01 & BQ110396 VD0107A01 & Versicolorin reductase & VDAG_00183 & $0.26^{*}$ & $0.30^{*}$ & $9.59^{* *}$ \\
\hline VD0104D02 & BQ110359 VD0107D07 & $\mathrm{H} 4 \mathrm{~N}$ reductase & VDAG_03665 & $0.15^{* *}$ & $0.19^{*}$ & $7.12^{* *}$ \\
\hline VD0103C12 & BQ110009 VD0101D02 & Laccase & VDAG_00189 & $0.26^{*}$ & $0.30^{*}$ & $8.49^{* *}$ \\
\hline VD0100C03 & BQ109845 VD0100C03 & Ayg1 & VDAG_04954 & 0.93 & 0.84 & $3.30^{*}$ \\
\hline \multirow[t]{2}{*}{ VD0106G02 } & BQ110282 VD0106G02; & ACE1 & - & $0.05^{* *}$ & $0.45^{* *}$ & $2.95^{* *}$ \\
\hline & VDAG_03150 & & & & & \\
\hline VD0104G06 & & Hypothetical & VDAG_07138 & $0.32^{* *}$ & $0.42^{* *}$ & $4.75^{* *}$ \\
\hline VD0103G07 & BQ109914 VD0100E03 & Hypothetical & VDAG_01806 & $0.21^{* *}$ & $0.23^{*}$ & $8.47^{* *}$ \\
\hline VD0108H01 & & Cytochrome p450 & VDAG_03650 & $0.42^{* *}$ & $0.60^{*}$ & $5.50^{* *}$ \\
\hline \multirow[t]{2}{*}{ VD0102H04 } & BQ110648 VD0202A11 & Hypothetical & VDAG_03287 & $0.03^{* *}$ & $0.63^{*}$ & $2.2^{*}$ \\
\hline & BQ109844 VD0100C02 & & & & & \\
\hline \multirow[t]{2}{*}{ VD0102H08 } & BQ110034 VD0102H08; & Acetoacetyl-CoA synthase & - & $0.11^{* *}$ & $0.61^{*}$ & $2.61^{*}$ \\
\hline & VDAG_09589 & & & & & \\
\hline \multicolumn{7}{|c|}{ Genes repressed in vdh1, LIQ } \\
\hline VD0103C06 & BQ110121 VD0103C06 & Fatty acid binding protein & - & $0.39^{* *}$ & $0.63^{* *}$ & 1.38 \\
\hline \multirow[t]{2}{*}{ VD0106H02 } & BQ110674 VD0206C06; & Heat shock protein & - & $0.61^{* *}$ & $0.58^{* *}$ & 2.234 \\
\hline & VDAG_04645 & & & & & \\
\hline VD0110G09 & BQ110535 VD0110G09 & Long chain base protein & VDAG_07480 & $0.51^{* *}$ & $0.70^{*}$ & 1.61 \\
\hline VD0103C11 & BQ110150 VD0103C11; & Oxidoreductase 2-nitropropane dioxygenase & VDAG_00590 & $0.56^{* *}$ & $0.65^{* *}$ & 1.60 \\
\hline VD0107C10 & BQ110341 VD0107C10; & Cellulose growth specific protein & VDAG_04795 & $0.33^{* *}$ & $0.79^{*}$ & 1.80 \\
\hline VD0102C03 & BQ109999 VD0102C03 & Phenazine biosynthesis protein & VDAG_00005 & $0.32^{* *}$ & $0.71^{* *}$ & 1.97 \\
\hline VD0105B02 & BQ110136 VD0105B02 & Vacuolar sorting associated & VDAG_03362 & $0.53^{* *}$ & $0.71^{* *}$ & 1.36 \\
\hline
\end{tabular}

${ }^{a}$ Control: wild-type strain Dvd-T5 grown on complete medium (CM)'agar; ${ }^{b} v d h 1: v d h 1$ strain (generated from wild type strain Dvd-T5 by targeted mutagenesis)

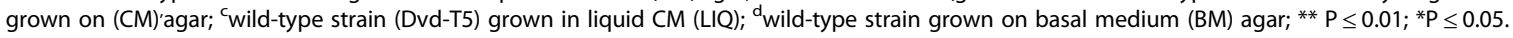

by both RNA-seq and RT-qPCR, but up-regulated by both microarray and Northern blot analyses, and VDAG_03150 which was, as mentioned above, not detected as differentially expressed by RNA-seq (Additional file 11) in strain VdLs.17 under the conditions tested (Additional file 9).

\section{Discussion}

Microsclerotia are critically important in the disease cycle of $V$. dahliae, and their development has been the subject of intense research for decades [6,12,13,17,18,36-38]. Though significant advances have been made in understanding the morphological and biochemical aspects of microsclerotia biogenesis, the molecular mechanisms underpinning the formation of these structures are still not well understood [39]. The aim of the current study was to identify genes that are differentially expressed in microsclerotia-producing (MS +) and NoMS cultures of $V$. dahliae, to shed further light on these molecular mechanisms. To conduct this work, we took advantage of genome-wide gene expression technologies (RNA-seq and microarray analyses), and also of the recently sequenced genome of $V$. dahliae strain VdLs.17 [19] for in silico functional annotation of the genes we identified as differentially expressed during MS development.

The RNA-seq analyses revealed a number of differentially expressed genes including those that encoded enzymes previously known to be involved in the fungal DHN-melanin biosynthetic pathway, including polyketide synthases, THN reductases, scytalone dehydratases, and laccases, [6,7,40,41]. Since MS maturation culminates with the synthesis and deposition of melanin granules in cell walls and intercellular spaces [37], the identification of the highly expressed genes encoding 
melanin biosynthetic-like enzymes indicated that the RNA-seq libraries were appropriate for evaluation of gene expression during MS maturation.

In the microarray analysis pigment biosynthesisencoding genes likewise formed the largest group of genes up-regulated during microsclerotial production, and showing high levels of expression, an expression pattern that was confirmed by Northern blot analyses of two of the putative melanin biosynthesis gene orthologs, VDAG_03393 and VDAG_00183. Moreover, pigment biosynthesis gene expression was similarly evident in an earlier EST study of $V$. dahliae cultures undergoing microsclerotia development [18]. Collectively, the overall consistency of these results signals the soundness of the experimental designs used herein.

Although the general biochemical scheme/pathway of fungal DHN melanin biosynthesis is well defined based on experimental evidence gleaned from genetic and biochemical studies [42], the exact identities, functions and multiplicity of all genes/enzymes involved in melanin biosynthesis, and the possible variation of function among species or strains has yet to be determined. The melanin synthesis pathway is complex, involving many regulatory, secretory and structural genes, a complexity that was made apparent by the identification of nine melanin synthesis-related genes that were detected in the RNA-seq study as highly expressed in the MS + culture. Some of these synthetic genes and putative regulators of melanin biosynthesis are clustered together in a $48.8 \mathrm{~kb}$ gene region of the $V$. dahliae genome. Similar melanin biosynthetic gene clusters are present in other fungi $[22,24,43]$, and such clusters often include one or more transcription factors, as found in this study.

The RT-qPCR analyses provided confirmation of differential gene expression between MS + and NoMS cultures, and further enabled analyses of gene expression at several culture time points. For example the known pigment biosynthesis gene VDAG_04954, encoding a protein highly similar to the melanin biosynthesis protein Ayg1 [44], the cytochrome p450 gene VDAG_03650, and the hypothetical protein-encoding gene VDAG_03287, were significantly down-regulated in the 6 month MS + culture, relative to the expression seen for the 12 day MS + culture. The down-regulation of VDAG_04954 in the six month $4^{\circ} \mathrm{C}$ culture may be indicative of completion of pigment synthesis. Similarly, the function of the cytochrome p450 (VDAG_03650) may not be required at this late stage.

Specific genes, such as VDAG_03650 and VDAG_ 04954, were identified by RT-qPCR analyses as being expressed in the $12 \mathrm{~d}$ MS + cultures at higher levels compared to that in the $7 \mathrm{~d} \mathrm{MS}+$ cultures. That this enhanced expression is suggestive of their roles in MS (survival structure) biogenesis is confirmed by the corresponding genes having previously been identified as ESTs (clones VD0108H01 and VD0100C03, respectively) expressed in DMS cultures [18], and also observed by microarray analysis to be highly expressed during microsclerotia development [20].

Morphological changes that occur during microsclerotia formation are well documented [10,37,38,45]. In the initial stage of MS formation, the hyphae aggregate, swell and form numerous septa. The septate cells enlarge and become spherical, forming clusters of cells by lateral budding, and during this process of MS maturation some cells undergo autolysis, resulting in a mixture, in individual MS, of dead and live cells [37]. The transition from vegetative (hyphal) growth to survival structure formation is expected to involve external and internal signals (morphogenetic triggers), as well as developmental reprogramming of molecular pathways that direct cell division, growth, and death. Intriguingly, the $V$. dahliae gene VDAG_00261, up-regulated 72-fold in the MS + culture, is highly similar to the Podospora anserina cell death-related gene IDI-3, the expression of which is induced during the vegetative incompatibility reaction [26]. Likewise, VDAG_01467 was up-regulated (13-fold) in the 10 day MS + culture, and is highly similar to the P. anserina gene Grg1, which exhibits increased expression in senescent cultures and has been implicated in control of lifespan [34]. Signaling and effector molecules that orchestrate the death of some cells during $V$. dahliae MS biogenesis may thus involve mechanisms similar to those that in other fungi control senescence and cell death during fungal vegetative incompatibility [46,47], with genes such as VDAG_00261 and VDAG_01467 involved in the developmental reprogramming of cells during MS maturation. However, the absence in the Verticillium group database of homologs of genes encoding other IDI proteins [26], as determined by BLASTP and tBLASTN (data not shown), suggests divergence between $P$. anserina and $V$. dahliae in the molecular mechanisms operating in stress responses and/or cell death control.

The process of sclerotia formation and maturation in filamentous fungi involves active translocation of molecules from hyphae to developing sclerotia, and exudation/excretion, from sclerotia to the cell environment, of various molecules such as water, amino acids, proteins, soluble carbohydrates, fatty acids, and enzymes [21]. Some of these molecules may be required for synthesis of storage compounds that act as energy reserves for sclerotia germination [21], and the observed increased expression in this study of certain transport protein genes, including those of $A B C$, MFS, and lipid transport systems, might reflect such a scenario.

Secreted carbohydrate-active enzymes (CAZys) may function not only in the disease process, but also in remodeling of fungal cell walls during MS maturation. The 
beta-1,3-glucanase encoded by VDAG_10470, which is up-regulated in the MS + library, may, for example, contribute to cell wall modifications that accompany morphological transitions in MS development, much as beta-1 ,3-glucanase is involved in morphogenesis in S. cerevisiae [48]. In addition, fungal periplasmic trehalases, which convert trehalose to glucose, are up-regulated under nutrient limiting or starvation conditions [49,50], and the observed enhanced expression, in the MS + library, of the putative periplasmic trehalase VDAG_03038 may therefore reflect depletion of nutrient levels in the actively growing 10-day $V$. dahliae cultures.

It is conceivable too that reduced expression of genes encoding specific cell wall-degrading activities may also be important for MS maturation. The endochitinase gene VDAG_08741 and the alpha-glucanase sequence VDAG_02431 (similar to S. pombe Agn1) were both down-regulated in the MS library. While the $S$. pombe protein degrades the septum, allowing cell separation [51], such separation may not be necessary in $V$. dahliae microsclerotia, which comprise both chains and clusters of spherical cells $[36,45]$. The chitin deacetylase encoded by VDAG_05660, which is up-regulated in the MS + library, may on the other hand protect the fungus by converting chitin to a de- $N$-acetylated product like that which has previously been shown to be unsuitable as a chitinase substrate [52]. A decrease in chitinolytic enzyme production during MS maturation, with a concomitant increase in cell wall protection, might be expected for production of the long-lived MS.

In addition to the new candidate genes identified by RNA-seq as differentially expressed in the MS library in this study, there was also a pattern of overlap with genes previously identified in the DMS EST library [18], and by the microarray analysis presented here (EMBL-EBI accession A-MEXP-2325). As described above, pigment biosynthesis genes formed one such clearly identifiable group, as did certain hypothetical/unknown genes. Notably, however, one of the hypothetical genes identified in both analyses (VDAG_03287) showed expression patterns that seem to be culture age dependent. This gene was shown by both microarray and Northern blot analyses to be up-regulated in 4-day old microsclerotiaproducing cultures, but by RNA-seq and RT-qPCR analysis was down-regulated in 10-day and 6 month microsclerotial cultures of VdLs.17. Likewise, the acetyl CoA synthase gene (VDAG_09589) was induced in the 4-day old cultures (as shown by microarray analysis), but not in the 10-day old cultures used to produce the RNA-seq libraries. Also, fluxes in the expression levels of certain other genes, like VDH1 [17], could have occurred at a developmental stage different than that sampled in the RNA-seq study. Such variation in results obtained through RNA-seq vs. microarray and previous
EST analyses may also reflect the difference in strains used for the studies (the lettuce isolate VdLs.17 and tomato isolate Dvd-T5, respectively), and/or the different culture conditions under which the fungi were grown prior to harvest (PDA overlaid with nitrocellulose membranes for RNA-seq analysis vs. cellulose membranes overlaid onto BM for microarray and EST analyses). While enhanced expression of cellulolytic enzymes was not expected to be observed in RNA-seq libraries prepared from cultures grown on nitrocellulose membranes, it is possible that starvation of the fungus in the presence of the cellulose may induce expression of genes encoding cell wall degrading enzymes, such as was observed for VDAG_04795 in the EST analysis [18], and in the present microarray analysis.

\section{Conclusion}

Information on the molecular control of microsclerotia development in $V$. dahliae is currently still sparse [39]. The developmental reprogramming from the normal growth state to survival structure (MS) formation is expected to involve external and internal signals (morphogenetic triggers) as well as unique cell signaling pathway (s), all of which are yet to be identified and elucidated. We envision that future functional characterization of the melanin synthesis and regulatory genes identified in this study, in conjunction with biochemical analyses, will help refine our understanding of the molecular basis of melanogenesis and MS morphogenesis in $V$. dahliae. Some of the genes identified as up- or down-regulated in the MS + vs. NoMS libraries may be involved in the morphogenetic processes that regulate different aspects of microsclerotial development. In addition, similar to the results obtained in previous EST analyses [18], approximately $50 \%$ of the genes that we identified by RNA-seq lacked significant similarity to any known genes. Functional analyses of some of these differentially expressed, unknown genes will undoubtedly advance our understanding of Verticillium development. The microsclerotia of $V$. dahliae have been considered important targets for disease control [10,11], and gene products identified in this study could potentially represent new targets for disease control through prevention of survival structure development.

\section{Methods}

\section{Fungal cultures for RNA-seq and RT-qPCR analyses}

Strain VdLs.17 of $V$. dahliae was initially grown on potato dextrose agar (PDA). A subculture was initiated by spreading conidia and hyphae onto $47 \mathrm{~mm}$ nitrocellulose membranes $(0.45 \mu \mathrm{m}$ pore size, Whatman, Maidstone, England), which were overlaid onto PDA plates. The cultures were maintained at $25^{\circ} \mathrm{C}$ in the dark. After $10 \mathrm{~d}$ cultures that formed dark microsclerotia (MS +), and those that did not form microsclerotia (NoMS) (Figure 1), were harvested for 
RNA-seq analyses (three replicates of each). The $10 \mathrm{~d}$ time point was selected based on the existing knowledge that in laboratory cultures of $V$. dahliae MS typically form over a span of 4 to 12 days. In some instances, however, such as in the $10 \mathrm{~d}$ NoMS cultures used for RNA-seq in this study, microsclerotia do not form. Additionally, cultures were harvested at different time points for analyses by RT-qPCR, including from a seven day-old culture with intermediate MS production, based on colony color (7 d MS +; such cultures become more darkly pigmented as the MS maturation process nears completion), a $12 \mathrm{~d}$ MS + culture, a $12 \mathrm{~d}$ NoMS culture, and 6-month-old MS cultures (6 mo $\mathrm{MS}+$ ) that had been maintained at $4^{\circ} \mathrm{C}$ for six months before harvesting (Figure 3). For each sample surface conidia were washed from the membranes using $2 \mathrm{ml}$ of deionized water and a cell spreader. For all RNA extractions the nitrocellulose membranes (Whatman) containing hyphae and microsclerotia, harvested at the appropriate time point, were ground to a fine powder in liquid nitrogen using a mortar and pestle.

\section{RNA-seq library preparation and construction}

Total RNA was extracted from the pulverized fungal tissue using a Qiagen RNeasy Kit (Valencia, CA) following the manufacturer's instructions, including an on-column DNase I digestion. The subsequent quality control steps and library construction were performed by Centrillion Biosciences (Palo Alto, CA). Briefly, total RNA quality was assessed using an Agilent 2100 bioanalyzer and an Agilent RNA 6000 Nano Kit (Agilent Technologies, Santa Clara, CA). All samples had RNA integrity numbers in the range of 6.8 to 7.6. The Ribo-Zero Magnetic Kit (Epicentre, Madison, Wisconsin) was used for an rRNA depletion step, and the samples were concentrated with an RNeasy MinElute Cleanup Kit (Qiagen). The RNA-Seq libraries were prepared with the ScriptSeq v2 RNA-seq Library Preparation Kit (Epicentre). Library validation was accomplished using Agilent DNA 1000 (Agilent Technologies) and Illumina Library Quantification (Kapa Biosystems, Woburn, MA) Kits. The DNA samples were sequenced using HiSeq 2000 (Illumina) at Centrillion Biosciences. The RNA-seq library statistics derived from the MS + and NoMS cultures are shown in Additional file 12.

\section{Bioinformatics and functional analyses}

Raw sequence reads in fastq format were aligned to the reference genome of the VdLs.17 strain of Verticillium dahliae (http://www.broadinstitute.org/annotation/genome/ verticillium_dahliae) following a workflow of Galaxy installed at the University of Alabama at Birmingham (http://galaxy.uabgrid.uab.edu). Pre-alignment was conducted to determine if end- trimming sequences reads was necessary, based upon read quality scores. The
BAM files were generated following RNA-seq workflow of tophat [53], cufflinks and cuffcompare [54]. These BAM files were loaded into Partek Genomics Suite 6.6 (Saint Louis, MO) for normalization and statistical analyses. Briefly, the reads per $\mathrm{kb}$ of exon model per million mapped (RPKM)-normalized reads [55] were calculated, and the expression levels of genes estimated [55-57]. Differential expression was determined by ANOVA, as described in the Partek Genomics Suite 6.6 instruction manual. Differentially expressed gene lists were then generated by applying a false discovery rate (FDR)-corrected cut off $\mathrm{P}$ value $(\mathrm{P})<0.05$.

For an initial genome-wide analysis (GWA), analyses of differential expression were conducted using untrimmed RNA-seq libraries with alignment to the $V$. dahliae transcriptome, and FDR $\mathrm{P}$ value correction. Those genes with $\mathrm{P}<0.05$ and $>1.3$ fold differences between libraries were considered significantly up- or down-regulated. A second GWA data set was prepared by RNA-seq analyses of the full $V$. dahliae transcriptome without FDR-correction. For this analysis, the last $10 \mathrm{bp}$ at the tail ends of the reads were trimmed based on a quality score of 30 . Those genes that were $>10$ fold upor down-regulated (82 and 21 genes, respectively) in the second GWA data set were reanalyzed along with various subsets of $V$. dahliae genes (Additional file 3), using a data-mining approach (DMA).

For the data-mining analysis (DMA), RNA-seq analyses were performed with trimmed sequence libraries and gene of interest (GOI) subsets, including those identified by the second GWA and having expression levels estimated as described above. With the exception of the PKSs, NRPSs, and hydrophobins, which were identified for this study using BLASTp analyses of the Verticillium Group Database, the GOI sets were characterized previously [19]. All of these sets are listed in GOI (Additional file 3) under the following categories: cytochrome p450s (P450s), LS region genes, Secretome, Major Facilitator Superfamily (MFS), ATP-Binding Cassette (ABC), Cysteine-Rich Proteins, and Vascular wilt-specific proteins. FDR correction was applied to the DMA, with FDR corrected $\mathrm{P}$ values $<0.05$ considered significant.

To attain additional insight on the functions of those differentially expressed genes encoding hypothetical proteins, Pfam domain searches were performed with protein sequence queries using a motif search (http://www.genome. jp/tools/motif/) and Pfam domain cutoff E-value 10e-6.

\section{RT-qPCR}

Verticillium dahliae strain VdLs. 17 was cultured at $25^{\circ} \mathrm{C}$ in the dark, for seven to twelve days, on nitrocellulose membranes (Whatman, Maidstone, England) overlaid onto PDA plates. To harvest, conidia were washed from membranes using $2 \mathrm{ml}$ deionized water and a cell 
spreader. Membranes were then ground in liquid nitrogen, and total RNA extracted from $100 \mathrm{mg}$ of the ground powder as described above for the RNA-seq library construction. After extraction, samples were further treated with TURBO DNase (Ambion, Austin, $\mathrm{TX})$ at $37^{\circ} \mathrm{C}$ for 30 minutes. RNA quality was checked using a Nanodrop (Thermo Scientific, Wilmington, $\mathrm{DE}$ ), and quantified using a Qubit Fluorometer (Invitrogen, Carlsbad, CA).

To identify an appropriate reference gene for $V$. dahliae MS + and NoMS cultures, the RNA-seq data were examined for potential reference genes for relative gene expression. Based on these analyses we determined that the expression of a ubiquitin gene (VDAG_05595) was not significantly up- or down-regulated in the MS + culture relative to that of the NoMS culture (Additional file 13). VDAG_05595 was therefore used as a reference for relative gene expression analyses in accordance with Pfaffl et al. [58]. Although beta-tubulin gene expression was approximately 2 -fold down-regulated in the 10 day MS + library, the use of the beta-tubulin gene in combination with VDAG_05595 as reference genes resulted in no changes in the significance $(\mathrm{P}<0.05)$ of the relative levels of expression for those genes that were classified as up- or down-regulated in the Taqman assays when VDAG_05595 was used as the only reference gene (data not shown).

The primers and Taqman probe combinations used in this study are listed in Additional file 14. Reverse transcription of $250 \mathrm{ng}$ RNA was performed in $20 \mu \mathrm{l}$ reactions, with a $5 \mathrm{~min}$. denaturation at $65^{\circ} \mathrm{C}$ and $0.5 \mu \mathrm{g}$ oligo-d(T)15 and $0.77 \mathrm{mM}$ dNTPs, followed by chilling on ice for at least five minutes, incubation at $55^{\circ} \mathrm{C}$ for 45 min with 200 U SuperScript III (Invitrogen), 1X first strand synthesis buffer, $5 \mathrm{mM}$ DTT and $40 \mathrm{U}$ RNAsin (Promega, Madison, WI), and incubation at $70^{\circ} \mathrm{C}$ for $15 \mathrm{~min}$ to inactivate reverse transcription. For the RTqPCR, $2 \mu \mathrm{l}$ cDNA was used as template, and reactions were done in 1X Gene Expression Master Mix (ABI; Applied Biosystems, Carlsbad, CA) with 1X Custom Taqman $^{\circ}$ Gene Expression Assay reagents (900 nM primer, $200 \mu \mathrm{M}$ Taqman probe) (ABI). Each sample was run in triplicate, as single probe reactions, in a LightCycler 480 II (Roche Molecular Diagnostics, Pleasanton, CA). The reaction profile included an initial $95^{\circ} \mathrm{C}, 10$ minute incubation followed by 40 amplification cycles: of $95^{\circ} \mathrm{C}$ for 15 seconds, and $60^{\circ} \mathrm{C}$ for 30 seconds. The resulting $\mathrm{Cq}$ values were analyzed for relative expression and errors were determined using REST software version 2009 [58]. PCR products were cloned into pCR 4.0-TOPO (Invitrogen) plasmid vector and sequenced to confirm primer specificity. The resulting plasmids, containing the gene sequences subsequently analyzed in Taqman assays, were used in a five-step, ten-fold dilution standard curve ( $1 \mathrm{ng}$ to $1 \mathrm{pg}$ ) to test the primer efficiency. All of the Taqman assays were at $\geq 100 \%$ efficiency (Additional file 14).

\section{Fungal cultures for microarray analyses}

The $V$. dahliae wild-type Dvd-T5 or the VDH1 mutant strain $(v d h 1)$ were grown on agar or in liquid media (BM and CM modified from that of Bennett and Lasure [59]). $\mathrm{CM}$ contained $1 \times$ nitrate salts $\left(\mathrm{NaNO}_{3}(6 \mathrm{~g} / \mathrm{L}), \mathrm{KCl}\right.$ (0.52 g/L), $\mathrm{MgSO}_{4} .7 \mathrm{H}_{2} \mathrm{O}(0.52 \mathrm{~g} / \mathrm{L}), \mathrm{KH}_{2} \mathrm{PO}_{4}(1.52 \mathrm{~g} / \mathrm{L}), 1 \times$ trace elements $\left(\mathrm{ZnSO}_{4} .7 \mathrm{H}_{2} \mathrm{O}(0.022 \mathrm{~g} / \mathrm{L}), \mathrm{H}_{3} \mathrm{BO}_{3}(0.011\right.$ $\mathrm{g} / \mathrm{L}), \mathrm{MnCl}_{2} .4 \mathrm{H}_{2} \mathrm{O}(0.005 \mathrm{~g} / \mathrm{L}), \mathrm{FeSO}_{4} .7 \mathrm{H}_{2} \mathrm{O}(0.005 \mathrm{~g} / \mathrm{L})$, $\mathrm{CoCl}_{2} .6 \mathrm{H} 2 \mathrm{O}(0.0017 \mathrm{~g} / \mathrm{L}), \mathrm{CuSO}_{4} .5 \mathrm{H}_{2} \mathrm{O}(0.0016 \mathrm{~g} / \mathrm{L})$, $\mathrm{Na}_{2} \mathrm{MoO}_{4} \cdot 2 \mathrm{H}_{2} \mathrm{O}(.0015 \mathrm{~g} / \mathrm{L})$, Na4EDTA $(0.05 \mathrm{~g} / \mathrm{L})$, glucose $(10 \mathrm{~g} / \mathrm{L})$, peptone $(2 \mathrm{~g} / \mathrm{L})$, yeast extract $(1 \mathrm{~g} / \mathrm{L})$, casamino acids $(1 \mathrm{~g} / \mathrm{L})$ and $1 \times$ vitamin solution (biotin, pyridoxine, thiamine, riboflavin, $p$-aminobenzoic acid, nicotinic acid, all at $0.01 \%(\mathrm{w} / \mathrm{v}))$. BM contained glucose $(10 \mathrm{~g} / \mathrm{L})$, sodium nitrate $(0.2 \mathrm{~g} / \mathrm{L}), 1 \times$ potassium salts $(\mathrm{KCl} 0.52 \mathrm{~g} / \mathrm{L}, \mathrm{MgSO} 4$ $.7 \mathrm{H} 2 \mathrm{O} 0.52 \mathrm{~g} / \mathrm{L}$, and $\left.\mathrm{KH}_{2} \mathrm{PO} 41.52 \mathrm{~g} / \mathrm{L}\right), 3 \mu \mathrm{M}$ thiamine $\mathrm{HCl}$, and $0.1 \mu \mathrm{M}$ biotin. Agar-grown cultures were inoculated by spreading $1 \times 10^{5}$ spores onto cellulose membranes (Research Products International, Mount Prospect, IL) overlaying the medium. To harvest the cells, the cellophane was lifted from the agar and immediately ground in liquid nitrogen. Liquid-grown cultures were centrifuged $(1480 \times g)$ for 10 minutes at $4^{\circ} \mathrm{C}$ to pellet spores and mycelial fragments. The supernatant was removed, and the pelleted fungal material was ground to a fine powder as above, in liquid nitrogen. Total RNA was isolated from the powdered tissue using TRIzol $^{\circ}$ Reagent (Invitrogen Canada Inc., Burlington, Canada) extraction as previously described [18].

\section{Microarray preparation and probe labeling}

cDNAs used for generating custom microarrays were selected from the $V$. dahliae simulated xylem fluid medium (SXM) and developing microsclerotia (DMS) libraries [18]. Individual cDNAs were amplified from the plasmid DNA by PCR, in $25 \mu \mathrm{L}$ reactions containing 400 $\mathrm{nM}$ universal primers $\mathrm{T} 3$ and $\mathrm{T} 7,50 \mathrm{ng}$ plasmid DNA, $200 \mu \mathrm{M}$ each dNTP, $2.5 \mathrm{mM} \mathrm{MgCl}_{2}, 200 \mathrm{mM}$ Tris- $\mathrm{HCl}$ (pH 8.4), $500 \mathrm{mM} \mathrm{KCl}$, and $0.5 \mathrm{U}$ Platinum Taq polymerase (Invitrogen, Canada Inc., Burlington, Canada). Denaturation of the template at $94^{\circ} \mathrm{C}$ for 2 minutes was followed by 30 amplification cycles $\left(94^{\circ} \mathrm{C}\right.$ for $45 \mathrm{sec}, 65^{\circ} \mathrm{C}$ for $45 \mathrm{sec}, 72^{\circ} \mathrm{C}$ for $1 \mathrm{~min}$ ), with a final $5 \mathrm{~min}$ extension period at $72^{\circ} \mathrm{C}$. To verify amplification, products were separated by electrophoresis through $0.8 \%$ agarose gels, and visualized by staining with $0.5 \mu \mathrm{g} / \mathrm{mL}$ ethidium bromide. Amplification reactions with low product yields were repeated using a $63^{\circ} \mathrm{C}$ annealing temperature, and amplification reactions resulting in non-specific 
amplification were repeated at an annealing temperature of $68^{\circ} \mathrm{C}$.

Amplification products were purified by filtration through Sephadex G50 columns (Amersham Biosciences, Baie d'Urfé, QC) in 96-well Polyfiltronics Unifilter plates (Fisher Scientific, Toronto, Canada), dried under vacuum at room temperature using a Savant Speedvac Plus SC210 (Global Medical Instrumentation, Ramsey, USA), and resuspended in $20 \mu \mathrm{L} \mathrm{50 \%} \mathrm{dimethyl} \mathrm{sulfoxide} \mathrm{at} \mathrm{a} \mathrm{final}$ cDNA concentration of approx $200 \mathrm{ng} / \mu \mathrm{L}$.

Arrays were constructed by printing amplified cDNA targets onto glass FMB cDNA slides (Full Moon BioSystems, Inc., Sunnyvale, CA), using the VP 470 Manual Glass Slide Indexing unit and VP 478 8-pin Glass Slide Replicator (V\&P Scientific, Inc. San Diego, CA). Each array consisted of 768 spots in an $18 \mathrm{~mm} \times 36 \mathrm{~mm}$ grid, with two adjacent duplicate spots representing each of 373 target genes. Following spotting, slides were air-dried, and DNA fixed to the slide by UV cross-linking.

To prepare labeled probes, total fungal RNA was further purified using the QIAquick RNeasy Kit (Qiagen) according to manufacturer's protocols. Ten $\mu \mathrm{g}$ purified total RNA was used as template in T7-RNA polymerase-catalyzed linear amplification reactions, using the RiboAmp RNA Amplification Kit (Arcturus, Mountainview, CA) according to supplier's directions. The resulting anti-sense RNA (aRNA) was quantified by UV spectrophotometry, and used to prepare probes.

Fluorescent dye-labeled probes, were generated by random-primed reverse transcription in the presence of cyanine-3 (Cy-3)-dCTP or cyanine-5 (Сy-5)-dCTP (Amersham Biosciences). Reverse transcription was done using $10 \mu \mathrm{g}$ RNA and the CyScribe First-Strand cDNA Labelling Kit (Amersham Biosciences), according to the manufacturer's directions. The CyScribe GFX Purification Kit (Amersham Biosciences) was used to remove unincorporated nucleotides from the $\mathrm{Cy}$-3- or $\mathrm{Cy}$-5-labelled probes, and fluorescent dye frequency of incorporation (FOI; picomoles of incorporated dye $x$ $(324.5 \div$ nanograms cDNA probe)) was measured by visible and UV spectrophotometry. Probes with FOIs between 20 and 50 were used in hybridizations.

\section{Microarray hybridization}

Pre-hybridization of slides was done for 20-30 minutes at $55^{\circ} \mathrm{C}$ in buffer containing $2 \times \mathrm{SSC}, 0.1 \% \mathrm{SDS}$, and $1 \%$ BSA. Following pre-hybridization slides were rinsed thoroughly with sterile distilled, deionized water (DDW), and dried using an ArrayIt microarray high-speed centrifuge (TeleChem International, Sunnyvale, CA).

For hybridization, 20 pmoles of each labeled cDNA probe were pooled and desiccated in an Eppendorf Vacufuge 5301 (Eppendorf, Mississauga Canada), re-suspended in $3 \mu \mathrm{L}$ nuclease-free DDW, and denatured at $90^{\circ} \mathrm{C}$ for
5 minutes. Thirty two $\mu \mathrm{L}$ FMB cDNA Hybridization buffer (Full Moon BioSystems, Inc.) was added to the probe solution (final volume $35 \mu \mathrm{L}$ ). The probe solution was applied to the slides, which were then covered with glass coverslips (Fisher Scientific), and placed in a humidified chamber at $42^{\circ} \mathrm{C}$, for 18 hours. Slides were then washed for $20 \mathrm{mi}-$ nutes in a $55^{\circ} \mathrm{C} 2 \times \mathrm{SSC} / 2 \%$ SDS solution, then three $\times$ $1 \mathrm{~min}$. in $55^{\circ} \mathrm{C} 0.2 \times \mathrm{SSC}$, quickly rinsed in DDW, and dried with a stream of filtered nitrogen gas.

Immediately after hybridization slides were scanned using a laser scanner (Virtek, Waterloo, ON) and processed, at the London Regional Genomics Institute at Robarts Research Institute (London, ON). The resulting raw scanned images of $\mathrm{Cy}-3$ and $\mathrm{Cy}-5$ fluorescence intensities were processed using Arrayvision 6.0 software (Imaging Research, St. Catharines, ON), wherein the local background was subtracted automatically from each spot.

Background-subtracted spot fluorescence values were imported into GeneSpring 6.0 software (Silicon Genetics, Redwood City, CA) for analysis, in which the intensities of replicate spots were averaged and used to calculate the expression ratios between the control and test conditions. Further normalization of the values was done using the Genespring Per Spot functions (data channel divided by control channel). Background filtering was not done, since the high background levels necessitated a high background cut-off that resulted in the elimination of a number of true positives (expression-validated genes; see below) from the analysis.

\section{Microarray data analysis and validation}

Data from duplicate spots and biological and technical replicates were pooled, and average expression values for the 373 genes were filtered to identify genes showing significant changes in gene expression (regulated genes) as determined using Student's $t$-test or ANOVA ( $\mathrm{p}<$ 0.01 ) with $\log _{2}$ of normalized values. Among the differentially regulated genes, 42 genes were identified whose expression changed 1.6-fold or more in at least one test condition when compared with the control condition.

Northern hybridization verification of microarray data was carried out using the DIG Filter Hybridization system (Roche Biomolecular Diagnostics), and methods recommended by the manufacturer. Briefly, total RNA $(8.5 \mu \mathrm{g})$ was electrophoretically separated on denaturing, $1 \%$ agarose, $2 \%$ formaldehyde gels. RNA was transferred by capillary blotting to positively charged nylon membranes (Roche Biomolecular Diagnostics), and fixed to the membrane by UV cross-linking.

DIG-labeled probes were prepared using as template $1 \mu \mathrm{g}$ EcoRI-linearized cDNA plasmids from the SXM or DMS libraries [18]. Thirty-minute pre-hybridizations and overnight hybridizations were done at $68^{\circ} \mathrm{C}$ in DIG Easy Hyb (Roche Molecular Diagnostics). High stringency washes 
were likewise done at $68^{\circ} \mathrm{C}$. Probe-target hybrids were visualized by chemiluminescent assay, using the chemiluminescent alkaline phosphatase substrate CSPD according to manufacturer's directions.

\section{Availability of supporting data}

RNA-seq data and experimental design were deposited in the ArrayExpress database at the European Molecular Biology Laboratory-European Bioinformatics Institute (EMBL-EBI) http://www.ebi.ac.uk/arrayexpress, with accession number E-MTAB-1581. Microarray data and experimental design were also deposited at EMBL-EBI, accession numbers E-MTAB-1793 and A-MEXP-2325, respectively.

\section{Additional files}

Additional file 1: Genes up-regulated in microsclerotia forming vs non microsclerotia forming culture of Verticillium dahliae as revealed by genome-wide analysis (approach 1 ) of RNA-seq data.

Additional file 2: Genes down-regulated in microsclerotia forming vs non microsclerotia forming culture of Verticillium dahliae as revealed by genome-wide analysis (approach 1) of RNA-seq data. Additional file 3: Data mining analyses (see file headings for additional details).

Additional file 4: Genes up-regulated in microsclerotia forming vs non microsclerotia forming cultures of Verticillium dahliae as revealed by data mining analysis of RNA-seq data.

Additional file 5: Genes down-regulated in microsclerotia forming vs. non microsclerotia forming cultures of Verticillium dahliae as revealed by data mining analysis of RNA-seq data.

Additional file 6: Comparison of fold changes of up-regulated genes from genome-wide analysis (GWA) and data mining analysis (DMA).

Additional file 7: Pie chart illustrating genes up- or down-regulated in the RNA-seq MS library.

Additional file 8: Comparison of fold changes observed in RT-qPCR and RNA-seq. The seven genes were identified by RNA-seq analyses as up- or down-regulated in cultures producing microsclerotia (MS +) or not producing microsclerotia (NoMS). A) Fold change for RT-qPCR was based on a comparison of 12 day MS cultures vs. 12 day NoMS cultures. B) comparison of 6 month MS + cultures vs 12 day NoMS cultures. The fold change values for RNA-seq were derived from the comparison of 10 day MS + cultures vs. 10 day NoMS cultures. Fold change is shown on a logarithmic scale.

Additional file 9: Verticillium dahliae strains and experimental parameters of the RNA-seq and microarray analyses.

Additional file 10: Validation of selected gene expression profiles by Northern hybridization analysis. RNA was isolated from complete medium (CM) agar-grown vdh1, liquid CM-grown WT, basal medium (BM) agar-grown WT, and CM agar-grown WT cultures. Blots were hybridized with DIG-labeled probes prepared from the CDNAs corresponding to the genes indicated. RNA quality and quantity was verified by ethidium bromide staining.

Additional file 11: Comparison of one or more of the four methods used to analyze gene expression.

Additional file 12: Statistics for RNA-Seq libraries derived from $V$. dahliae cultures producing microsclerotia (MS +) or not producing microsclerotia (NoMS).

Additional file 13: RNA-seq data for reference gene selection.
Additional file 14: Primers and Taqman probes used in RT-qPCR in this study.

Competing interests

The authors declare that they have no competing interests.

Authors' contributions

Wrote the manuscript: SJK, DD; conceived and designed RNA-seq experiments: SJK, DD, DC; conceived and designed RT-qPCR experiments: $\mathrm{SJK}, \mathrm{AA}$; conceived and designed microarray and Northern blot experiments: KFD, AK; Identification of genes involved in secondary metabolism: MGP, SJK; data analyses: SJK, DD, MGP, DC, AA, AK, KFD. All authors approved the final version of the manuscript.

\section{Acknowledgements}

The authors acknowledge funding from the California Department of Food and Agriculture, Agreement SCB09023, and Natural Sciences and Engineering Research Council of Canada. We are thankful for the help of Patrick

Chapman for contributions in tabulating microarray data for database submission.

Mention of trade names or commercial products in this publication is solely for the purpose of providing specific information and does not imply recommendation or endorsement by the United States Department of Agriculture (USDA). USDA is an equal opportunity provider and employer.

\section{Author details}

'United States Department of Agriculture - Agricultural Research Service, Salinas, CA, USA. ${ }^{2}$ Comprehensive Cancer Center \& Division of Preventive Medicine, University of Alabama at Birmingham, Birmingham, AL, USA. ${ }^{3}$ Department of Biology, University of Western Ontario, London, ON, Canada. ${ }^{4}$ Agriculture and Agri-Food Canada, London, ON, Canada. ${ }^{5}$ Department of Physiological and Biological Science, Western New England University, Springfield, MA, USA. ${ }^{6}$ Instituto de Hortofruticultura Subtropical y Mediterránea "La Mayora"-Consejo Superior de Investigaciones Científicas (IHSM-UMA-CSIC), Estación Experimental "La Mayora", Algarrobo-Costa, Málaga, Spain.

Received: 8 March 2013 Accepted: 4 September 2013

Published: 9 September 2013

\section{References}

1. Klosterman SJ, Atallah ZK, Vallad GE, Subbarao KV: Diversity, pathogenicity, and management of Verticillium species. Ann Rev Phytopathol 2009, 47:39-62.

2. Pegg GF, Brady BL: Verticillium Wilts. Wallingford: CABI Publishing; 2002

3. Wilhelm S: Longevity of the Verticillium wilt fungus in the laboratory and field. Phytopathology 1955, 45:180-181.

4. Schreiber LR, Green RJ: Effect of root exudates on germination of conidia and microsclerotia of Verticillium albo-atrum inhibited by the soil fungistatic principle. Phytopathology 1963, 53:260-264.

5. Fitzell R, Evans G, Fahy PC: Studies on the colonization of plant roots by Verticillium dahliae Klebahn with use of immunofluorescent staining. Austr J Bot 1980, 28:357-368.

6. Butler MJ, Day AW: Fungal melanins: a review. Can J Microbiol 1998, 44:1115-1136

7. Wheeler $\mathrm{MH}$ : Comparisons of fungal melanin biosynthesis in ascomycetous, imperfect and basidiomycetous fungi. Trans Br Mycological Soc 1983, 81:29-36.

8. Bell $A A$, Wheeler $M H$ : Biosynthesis and functions of fungal melanins. Ann Rev Phytopathol 1986, 24:411-451.

9. Bell AA, Puhalla JE, Tolmsoff WJ, Stipanovic RD: Use of mutants to establish (+)-scytalone as an intermediate in melanin biosynthesis by Verticillium dahliae. Can J Microbiol 1976, 22:787-799.

10. Gordee RS, Porter CL: Structure, germination and physiology of microsclerotia of Verticillium albo-atrum. Mycologia 1961, 53:171-182.

11. Debode J, Maeyer KD, Perneel M, Pannecoucque J, Backer GD, Höfte M: Biosurfactants are involved in the biological control of Verticillium microsclerotia by Pseudomonas spp. J Appl Microbiol 2007, 103:1184-1196. 
12. Hawke MA, Lazarovits G: Production and manipulation of individual microsclerotia of Verticillium dahliae for use in studies of survival. Phytopathology 1994, 84:883-890.

13. Coley-Smith JR, Cooke RC: Survival and germination of fungal sclerotia. Ann Rev Phytopathol 1971, 9:65-92.

14. Gao F, Zhou B-J, Li G-Y, Jia P-S, Li H, Zhao Y-L, Zhao P, Xia G-X, Guo H-S: A glutamic acid-rich protein identified in Verticillium dahliae from an insertional mutagenesis affects microsclerotial formation and pathogenicity. PLOS ONE 2010, 5:e15319.

15. Maruthachalam K, Klosterman SJ, Kang S, Hayes RJ, Subbarao KV: Identification of pathogenicity-related genes in the vascular wilt fungus Verticillium dahliae by Agrobacterium tumefaciens-mediated T-DNA insertional mutagenesis. Mol Biotechnol 2011, 49:209-221.

16. Tzima AK, Paplomatas EJ, Rauyaree P, Kang S: Roles of the catalytic subunit of cAMP-dependent protein kinase $A$ in virulence and development of the soilborne plant pathogen. Fungal Genet Biol 2010, 7:406-415.

17. Klimes A, Dobinson KF: A hydrophobin gene, $V D H 1$, is involved in microsclerotial development and spore viability in the plant pathogen Verticillium dahliae. Fungal Genet Biol 2006, 43:283-294.

18. Neumann MJ, Dobinson KF: Sequence tag analysis of gene expression during pathogenic growth and microsclerotia development in the vascular wilt pathogen Verticillium dahliae. Fungal Genet Biol 2003, 38:54-62.

19. Klosterman SJ, Subbarao KV, Kang S, Veronese P, Gold SE, Thomma BPHJ, Chen Z, Henrissat B, Lee Y-H, Park J, Garcia-Pedrajas MD, Barbara DJ, Anchieta A, de Jonge R, Santhanam P, Maruthachalam K, Atallah Z, Amyotte SG, Paz Z, Inderbitzin P, Hayes RJ, Heiman DI, Young S, Zeng Q, Engels R, Galagan J, Cuomo CA, Dobinson KF, Ma L-J: Comparative genomics yields insights into niche adaptation of plant vascular wilt pathogens PLoS Pathog 2011, 7:e1002137.

20. Klimes A: Identification and characterization of genes associated with microsclerotial development in the plant pathogen Verticillium dahliae. PhD Thesis 2006. University of Western Ontario

21. Chet I, Henis Y: Sclerotial morphogenesis in fungi. Ann Rev Phytopathol 1975, 13:169-192.

22. Eliahu N, Igbaria A, Rose MS, Horwitz BA, Lev S: Melanin biosynthesis in the maize pathogen Cochliobolus heterostrophus depends on two mitogen-activated protein kinases, Chk1 and Mps1, and the transcription factor Cmr1. Eukaryot Cell 2007, 6:421-429.

23. Tsuji G, Kenmochi Y, Takano Y, Sweigard J, Farrall L, Furusawa I, Horino O, Kubo Y: Novel fungal transcriptional activators, Cmr1p of Colletotrichum lagenarium and Pig1p of Magnaporthe grisea, contain Cys2His2 zinc finger and Zn(II)2Cys6 binuclear cluster DNA-binding motifs and regulate transcription of melanin biosynthesis genes in a developmentally specific manner. Mol Microbiol 2000, 38:940-954.

24. Woo PCY, Tam EWT, Chong KTK, Cai JJ, Tung ETK, Ngan AHY, Lau SKP, Yuen $\mathrm{K}-\mathrm{Y}$ : High diversity of polyketide synthase genes and the melanin biosynthesis gene cluster in Penicillium marneffei. FEBS J 2010 277:3750-3758.

25. Yu J, Chang P-K, Ehrlich KC, Cary JW, Montalbano B, Dyer JM, Bhatnagar D, Cleveland TE: Characterization of the critical amino acids of an Aspergillus parasiticus cytochrome $\mathrm{P}-450$ monooxygenase encoded by ord $A$ that is involved in the biosynthesis of aflatoxins B1, G1, B2, and G2. Appl Environ Microbiol 1998, 64:4834-4841.

26. Bourges N, Groppi A, Barreau C, Clave C, Begueret J: Regulation of gene expression during the vegetative incompatibility reaction in Podospora anserina: Characterization of three induced genes. Genetics 1998, 150:633-641.

27. Dementhon K, Paoletti M, Pinan-Lucarre B, Loubradou-Bourges N, Sabourin $M$, Saupe SJ, Clave C: Rapamycin mimics the incompatibility reaction in the fungus Podospora anserina. Eukaryot Cell 2003, 2:238-246.

28. Puyesky M, Benhamou N, Noyola PP, Bauw G, Ziv T, Van Montagu M, Herrera-Estrella A, Horwitz BA: Developmental regulation of $\mathrm{cmp1}$, a gene encoding a multidomain conidiospore surface protein of Trichoderma. Fungal Genet Biol 1999, 27:88-99.

29. Walker SK, Garrill A: Actin microfilaments in fungi. Mycologist 2006, 20:26-31.

30. Feoktistova A, McCollum D, Ohi R, Gould KL: Identification and characterization of Schizosaccharomyces pombe asp1+, a gene that interacts with mutations in the Arp2/3 complex and actin. Genetics 1999 152:895-908.
31. Mouassite M, Camougrand N, Schwob E, Demaison G, Laclau M, Guérin M: The 'SUN' family: yeast SUN4/SCW3 is involved in cell septation. Yeast 2000, 16:905-919.

32. Robbertse B, Yoder OC, Nguyen A, Schoch CL, Turgeon BG: Deletion of all Cochliobolus heterostrophus monofunctional catalase-encoding genes reveals a role for one in sensitivity to oxidative stress but none with a role in virulence. Mol Plant-Microbe Interact 2003, 16:1013-1021.

33. Papapostolou I, Georgiou CD: Hydrogen peroxide is involved in the sclerotial differentiation of filamentous phytopathogenic fungi. J Appl Microbiol 2010, 109:1929-1936.

34. Kimpel E, Osiewacz HD: PaGrg1, a glucose-repressible gene of Podospora anserina that is differentially expressed during lifespan. Curr Genet 1999, 35:557-563.

35. Walther TC, Brickner JH, Aguilar PS, Bernales S, Pantoja C, Walter P. Eisosomes mark static sites of endocytosis. Nature 2006, 439:998-1003.

36. Brandt WH: Morphogenesis in Verticillium: Effect of light and ultraviolet radiation on microsclerotia and melanin. Can J Bot 1964, 42:1017-1023.

37. Griffiths DA: The fine structure of developing microsclerotia of Verticillium dahliae Kleb. Arch Microbiol 1970, 74:207-212.

38. Klimes A, Amyotte SG, Grant S, Kang S, Dobinson KF: Microsclerotia development in Verticillium dahliae: Regulation and differential expression of the hydrophobin gene VDH1. Fungal Genet Biol 2008, 45:1525-1532.

39. Fradin EF, Thomma BPHJ: Physiology and molecular aspects of Verticillium wilt diseases caused by $V$. dahliae and V. albo-atrum. Mol Plant Pathol 2006, 7:71-86

40. Thompson JE, Fahnestock S, Farrall L, Liao D-I, Valent B, Jordan DB: The second naphthol reductase of fungal melanin biosynthesis in Magnaporthe grisea: tetrahydroxynaphthalene reductase. J Biol Chem 2000, 275:34867-34872.

41. Butler MJ, Gardiner RB, Day AW: Melanin synthesis by Sclerotinia sclerotiorum. Mycologia 2009, 101:296-304.

42. Henson JM, Butler MJ, Day AW: The dark side of the mycelium: Melanins of phytopathogenic fungi. Ann Rev Phytopathol 1999, 37:447-471.

43. Tsai H-F, Wheeler MH, Chang YC, Kwon-Chung KJ: A developmentally regulated gene cluster involved in conidial pigment biosynthesis in Aspergillus fumigatus. J Bacteriol 1999, 181:6469-6477.

44. Fujii I, Yasuoka Y, Tsai H-F, Chang YC, Kwon-Chung KJ, Ebizuka Y: Hydrolytic polyketide shortening by ayg1p, a novel enzyme involved in fungal melanin biosynthesis. J Biol Chem 2004, 279:44613-44620.

45. Karapapa VK, Bainbridge BW, Heale JB: Morphological and molecular characterization of Verticillium longisporum comb. nov., pathogenic to oilseed rape. Mycol Res 1997, 101:1281-1294.

46. Dementhon K, lyer G, Glass NL: VIB-1 is required for expression of genes necessary for programmed cell death in Neurospora crassa. Eukaryot Cell 2006, 5:2161-2173

47. Paoletti $\mathrm{M}$, Clave $\mathrm{C}$ : The fungus-specific HET domain mediates programmed cell death in Podospora anserina. Eukaryot Cell 2007, 6:2001-2008.

48. Baladron V, Ufano S, Dueñas E, Martín-Cuadrado AB, del Rey F, Vazquez de Aldana CR: Eng1p, an endo-1,3- $\beta$-glucanase localized at the daughter side of the septum, is involved in cell separation in Saccharomyces cerevisiae. Eukaryot Cell 2002, 1:774-786.

49. Jorge JA, Polizeli ML, Thevelein JM, Terenzi HF: Trehalases and trehalose hydrolysis in fungi. FEMS Microbiol Lett 1997, 154:165-171.

50. Parrou JL, Jules M, Beltran G, François J: Acid trehalase in yeasts and filamentous fungi: Localization, regulation and physiological function. FEMS Yeast Res 2005, 5:503-511.

51. Dekker N, Speijer D, Grun CH, van den Berg M, de Haan A, Hochstenbach F: Role of the alpha-glucanase Agn1p in fission-yeast cell separation. Mol Biol Cell 2004, 15:3903-3914.

52. Blair DE, Hekmat O, Schuttelkopf W, Shrestha B, Tokuyasu K, Withers SG, van Aalten DMF: Structure and mechanism of chitin deacetylase from the fungal pathogen Colletotrichum lindemuthianum. Biochemistry 2006 45:9416-9426

53. Trapnell C, Pachter L, Salzberg SL: TopHat: discovering splice junctions with RNA-Seq. Bioinformatics 2009, 25:1105-1111.

54. Trapnell C, Williams BA, Pertea G, Mortazavi A, Kwan G, van Baren MJ, Salzberg SL, Wold BJ, Pachter L: Transcript assembly and quantification by RNA-Seq reveals unannotated transcripts and isoform switching during cell differentiation. Nat Biotechnol 2010, 28:511-515. 
55. Mortazavi A, Williams BA, McCue K, Schaeffer L, Wold BJ: Mapping and quantifying mammalian transcriptomes by RNA-Seq. Nat Methods 2008, 5:621-628.

56. Xing Y, Yu T, Wu YN, Roy M, Kim J, Lee C: An expectation-maximization algorithm for probabilistic reconstructions of full-length isoforms from splice graphs. Nucleic Acids Res 2006, 34:3150-3160.

57. Wang ET, Sandberg R, Luo S, Khrebtukova I, Zhang L, Mayr C, Kingsmore SF, Schroth GP, Burge CB: Alternative isoform regulation in human tissue transcriptomes. Nature 2008, 456:470-476.

58. Pfaffl MW, Horgan GW, Dempfle L: Relative expression software tool (RESTO) for group-wise comparison and statistical analysis of relative expression results in real-time PCR. Nucleic Acids Res 2002, 30:e36.

59. Bennett JW, Lasure LL: Growth media. San Diego: Academic; 1991.

doi:10.1186/1471-2164-14-607

Cite this article as: Duressa et al:: RNA-seq analyses of gene expression in the microsclerotia of Verticillium dahliae. BMC Genomics 2013 14:607.

\section{Submit your next manuscript to BioMed Central and take full advantage of:}

- Convenient online submission

- Thorough peer review

- No space constraints or color figure charges

- Immediate publication on acceptance

- Inclusion in PubMed, CAS, Scopus and Google Scholar

- Research which is freely available for redistribution 